\title{
Cortical abnormalities in adults and adolescents with major depression based on brain scans from 20 cohorts worldwide in the ENIGMA Major Depressive Disorder Working Group
}

\author{
L Schmaal ${ }^{1}$, DP Hibar ${ }^{2}$, PG Sämann ${ }^{3}$, GB Hall ${ }^{4}$, BT Baune ${ }^{5}$, N Jahanshad ${ }^{2}$, JW Cheung ${ }^{2}$, TGM van Erp ${ }^{6}$, D Bos ${ }^{7,8}$, MA Ikram, ${ }^{7,8,9}$, \\ MW Vernooij ${ }^{7,}$, WJ Niessen ${ }^{7,10,11}$, H Tiemeier $^{8,12}$ A Hofman $^{8}$, K Wittfeld $^{13}$, HJ Grabe ${ }^{13,14}$, D Janowitz ${ }^{14}$, R Bülow ${ }^{15}$, M Selonke ${ }^{14}$, \\ H Völzke ${ }^{16,17,18}$, D Grotegerd ${ }^{19}$, U Dannlowski ${ }^{19,20}$, V Arolt ${ }^{19}$, N Opel $^{19}$, W Heindel ${ }^{21}$, H Kugel $^{21}$, D Hoehn ${ }^{3}$, M Czisch ${ }^{3}$,

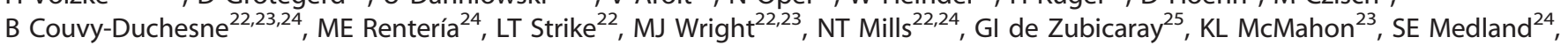 \\ NG Martin ${ }^{24}$, NA Gillespie ${ }^{26}$, R Goya-Maldonado ${ }^{27}$, O Gruber ${ }^{28}$, B Krämer ${ }^{28}$, SN Hatton ${ }^{29}$, J Lagopoulos ${ }^{29}$, IB Hickie ${ }^{29}$, T Frodl ${ }^{30,31}$, \\ A Carballedo ${ }^{31}$, EM Frey ${ }^{32}$, LS van Velzen ${ }^{1}$, BWJH Penninx ${ }^{1}$, M-J van Tol ${ }^{33}$, NJ van der Wee ${ }^{34}$, CG Davey ${ }^{35,36,37}$, BJ Harrison ${ }^{37}$, B Mwangi $^{38}$, \\ B Cao ${ }^{38}$, JC Soares ${ }^{38}$, IM Veer ${ }^{39}$, H Walter $^{39}$, D Schoepf ${ }^{40}$, B Zurowski $^{41}$, C Konrad $^{20,42}$, E Schramm $^{43}$, C Normann $^{43}, \mathrm{~K} \mathrm{Schnell}^{28}$,

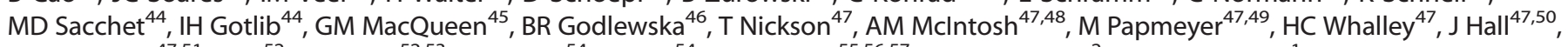 \\ JE Sussmann ${ }^{47,51}, \mathrm{M} \mathrm{Li}^{52}$, M Walter ${ }^{52,53}$, L Aftanas ${ }^{54}$, I Brack ${ }^{54}$, NA Bokhan ${ }^{55,56,57}$, PM Thompson ${ }^{2}$ and DJ Veltman ${ }^{1}$ for the ENIGMA-Major \\ Depressive Disorder Working Group ${ }^{58}$
}

The neuro-anatomical substrates of major depressive disorder (MDD) are still not well understood, despite many neuroimaging studies over the past few decades. Here we present the largest ever worldwide study by the ENIGMA (Enhancing Neuro Imaging Genetics through Meta-Analysis) Major Depressive Disorder Working Group on cortical structural alterations in MDD. Structural T1-weighted brain magnetic resonance imaging (MRI) scans from 2148 MDD patients and 7957 healthy controls were analysed with harmonized protocols at 20 sites around the world. To detect consistent effects of MDD and its modulators on cortical thickness and surface area estimates derived from MRI, statistical effects from sites were meta-analysed separately for adults and adolescents.

${ }^{1}$ Department of Psychiatry and Neuroscience Campus Amsterdam, VU University Medical Center, Amsterdam, The Netherlands; ${ }^{2}$ Imaging Genetics Center, Mark and Mary Stevens Neuroimaging and Informatics Institute, Keck School of Medicine of USC, Marina del Rey, CA, USA; ${ }^{3}$ Neuroimaging Core Unit, Max Planck Institute of Psychiatry, Munich, Germany; ${ }^{4}$ Department of Psychology, Neuroscience and Behaviour, McMaster University, Hamilton, ON, Canada; ${ }^{5}$ Discipline of Psychiatry, University of Adelaide, Adelaide, SA, Australia; ${ }^{6}$ Department of Psychiatry and Human Behavior, University of California, Irvine, CA, USA; ${ }^{7}$ Department of Radiology, Erasmus MC University Medical Center, Rotterdam, The Netherlands; ${ }^{8}$ Department of Epidemiology, Erasmus MC University Medical Center, Rotterdam, The Netherlands; ${ }^{9}$ Department of Neurology, Erasmus MC University Medical Center, Rotterdam, The Netherlands; ${ }^{10}$ Department of Medical Informatics, Erasmus MC University Medical Center, Rotterdam, The Netherlands; ${ }^{11}$ Faculty of Applied Sciences, Delft University of Technology, Delft, The Netherlands; ${ }^{12}$ Department of Child and Adolescent Psychiatry, Erasmus University Medical Center-Sophia Children's Hospital, Rotterdam, The Netherlands; ${ }^{13}$ German Center for Neurodegenerative Diseases (DZNE), Site Rostock/Greifswald, Germany; ${ }^{14}$ Department of Psychiatry and Psychotherapy, University Medicine Greifswald, Greifswald, Germany; ${ }^{15}$ Institute for Diagnostic Radiology and Neuroradiology, University Medicine Greifswald, Greifswald, Germany; ${ }^{16}$ Institute for Community Medicine, University Medicine Greifswald, Greifswald, Germany; ${ }^{17}$ German Center for Cardiovascular Research (DZHK), partner site Griefswald, Greifswald, Germany; ${ }^{18}$ German Center for Diabetes Research (DZD), partner site Griefswald, Greifswald, Germany; ${ }^{19}$ Department of Psychiatry, University of Muenster, Muenster, Germany; ${ }^{20}$ Department of Psychiatry, University of Marburg, Marburg, Germany; ${ }^{21}$ Department of Clinical Radiology, University of Muenster, Muenster, Germany; ${ }^{22}$ Queensland Brain Institute, The University of Queensland, Brisbane, QLD, Australia; ${ }^{23}$ Center for Advanced Imaging, The University of Queensland, Brisbane, QLD, Australia; ${ }^{24}$ Queensland Institute of Medical Research Berghofer, Brisbane, QLD, Australia; ${ }^{25}$ Institute of Health and Biomedical Innovation, Queensland University of Technology, Brisbane, QLD, Australia; ${ }^{26}$ Department of Psychiatry, Virginia Institute for Psychiatric and Behavioral Genetics, Richmond, VA, USA; ${ }^{27}$ Centre for Translational Research in Systems Neuroscience and Psychiatry, Department of Psychiatry and Psychotherapy, University Medical Center (UMG), Georg-August-University, Göttingen, Germany; ${ }^{28}$ Section for Experimental Psychopathology and Neuroimaging, Department of General Psychiatry, Heidelberg University Hospital, Heidelberg, Germany; ${ }^{29}$ Clinical Research Unit, Brain and Mind Centre, University of Sydney, Camperdown, NSW, Australia; ${ }^{30}$ Department of Psychiatry and Psychotherapy, Otto von Guericke University, Magdeburg, Germany; ${ }^{31}$ Department of Psychiatry and Institute of Neuroscience, Trinity College, Dublin, Ireland; ${ }^{32}$ Department of Psychiatry and Psychotherapy, University of Regensburg, Regensburg, Germany; ${ }^{33}$ Neuroimaging Center, Section of Cognitive Neuropsychiatry, Department of Neuroscience, University Medical Center Groningen, University of Groningen, Groningen, The Netherlands; ${ }^{34}$ Department of Psychiatry and Leiden Institute for Brain and Cognition, Leiden University Medical Center, Leiden, The Netherlands; ${ }^{35}$ Orygen, The National Centre of Excellence in Youth Mental Health, Melbourne, VIC, Australia; ${ }^{36}$ Centre for Youth Mental Health, The University of Melbourne, Melbourne, VIC, Australia; ${ }^{37}$ Melbourne Neuropsychiatry Centre, Department of Psychiatry, The University of Melbourne, Melbourne, VIC, Australia; ${ }^{38}$ UT Center of Excellence on Mood Disoders, Department of Psychiatry and Behavioral Sciences, University of Texas Health Science Center at Houston, Houston, TX, USA; ${ }^{39}$ Department of Psychiatry and Psychotherapy, Charité Universitätsmedizin Berlin, Berlin, Germany; ${ }^{40}$ Department of Psychiatry and Psychotherapy, University of Bonn, Bonn, Germany; ${ }^{41}$ Center for Integrative Psychiatry, University of Lübeck, Lübeck, Germany; ${ }^{42}$ Department of Psychiatry and Psychotherapy, Agaplesion Diakonieklinikum Rotenburg, Rotenburg, Germany; ${ }^{43}$ Department of Psychiatry and Psychotherapy, University Medical Center Freiburg, Freiburg, Germany; ${ }^{44}$ Neurosciences Program and Department of Psychology, Stanford University, Stanford, CA, USA; ${ }^{45}$ Department of Psychiatry, University of Calgary, Calgary, AB, Canada; ${ }^{46}$ University Department of Psychiatry, Warneford Hospital, Oxford, UK; ${ }^{47}$ Division of Psychiatry, University of Edinburgh, Royal Edinburgh Hospital, Edinburgh, UK; ${ }^{48}$ Centre for Cogntive Ageing and Cogntive Epidemiology, University of Edinburgh, Edinburg, UK; ${ }^{49}$ Division of Systems Neuroscience of Psychopathology, Translational Research Center, University Hospital of Psychiatry, University of Bern, Bern, Switzerland; ${ }^{50}$ Neuroscience and Mental Health Research Institute, Cardiff University, Cardiff, UK; ${ }^{51}$ Department of Psychiatry, NHS Borders, Melrose, UK; ${ }^{52}$ Leibniz Institute for Neurobiology, Magdeburg, Germany; ${ }^{53}$ Department of Psychiatry, University Tübingen, Tübingen, Germany; ${ }^{54}$ Department of Experimental and Clinical Neuroscience, Scientific Research Institute of Physiology and Basic Medicine, Novosibirsk, Russia; ${ }^{55}$ Mental Health Research Institute, Tomsk, Russia; ${ }^{56} \mathrm{Faculty}$ of Psychology, National Research Tomsk State University, Tomsk, Russia and ${ }^{57}$ Department of General Medicine, Siberian State Medical University, Tomsk, Russia. Correspondence: Dr L Schmaal, Department of Psychiatry and Neuroscience Campus Amsterdam, GGZ inGeest/ VU University Medical Center, P.O. Box 74077, Amsterdam 1070 BB, The Netherlands.

E-mail: I.schmaal@ggzingeest.nl

${ }^{58}$ The ENIGMA-Major Depressive Disorder Working Groups are available at: http://enigma.ini.usc.edu/ongoing/enigma-mdd-working-group/.

Received 20 January 2016; revised 25 February 2016; accepted 17 March 2016; published online 3 May 2016 
Adults with MDD had thinner cortical gray matter than controls in the orbitofrontal cortex (OFC), anterior and posterior cingulate, insula and temporal lobes (Cohen's $d$ effect sizes: -0.10 to -0.14 ). These effects were most pronounced in first episode and adultonset patients (> 21 years). Compared to matched controls, adolescents with MDD had lower total surface area (but no differences in cortical thickness) and regional reductions in frontal regions (medial OFC and superior frontal gyrus) and primary and higherorder visual, somatosensory and motor areas $(d:-0.26$ to -0.57$)$. The strongest effects were found in recurrent adolescent patients. This highly powered global effort to identify consistent brain abnormalities showed widespread cortical alterations in MDD patients as compared to controls and suggests that MDD may impact brain structure in a highly dynamic way, with different patterns of alterations at different stages of life.

Molecular Psychiatry (2017) 22, 900-909; doi:10.1038/mp.2016.60; published online 3 May 2016

\section{INTRODUCTION}

Major depressive disorder (MDD) is the single most common psychiatric disorder, affecting approximately 350 million people each year. ${ }^{1}$ Even so, its pathogenesis and profile of effects in the brain are still not clear. Therefore, in 2013, we initiated the MDD Working Group within the Enhancing Neuro Imaging Genetics through Meta-Analysis (ENIGMA) consortium (http://enigma.ini. usc.edu/) in which researchers around the world collaborate to boost statistical power to elucidate brain abnormalities in MDD. Recently, we reported subcortical volume differences between MDD patients and healthy controls that were related to clinical characteristics, based on data from 8927 individuals using an individual participant data-based meta-analysis approach. Subcortical volume differences were the greatest in the hippocampus, with the strongest effects in recurrent or early-onset patients. ${ }^{2}$ Here we present results on cortical structural differences in an even larger sample $(N=10105)$.

With regard to cortical structural abnormalities in MDD, prior magnetic resonance imaging (MRI) studies, summarized in retrospective meta-analyses of individually published works, mainly implicate the (para)limbic circuitry, including dorsomedial prefrontal cortex (PFC), orbitofrontal cortex (OFC) and (rostral) anterior cingulate cortex (ACC), albeit with large variability across studies. ${ }^{3-7}$ Findings are inconclusive regarding the temporal and lateral PFC. ${ }^{4,8}$ Inconsistencies arise owing to differences in scanning and analysis methods, the limited power to detect subtle effects in small samples and clinical variations in medication status, ${ }^{6}$ lifetime disease burden, ${ }^{6}$ age at disease onset ${ }^{9}$ and adult vs adolescent study samples. ${ }^{10}$

Differences in data acquisition protocols and processing and differences in statistical analyses performed are a key source of heterogeneity. For example, different techniques for assessing morphometric deficits in MDD are used. Many studies use automated MRI analyses such as voxel-based morphometry, ${ }^{11}$ which avoid labour-intensive manual tracings and improve reproducibility. Others use surface-based methods that generate detailed maps of cortical thickness and surface area, which may differ in their underlying cellular mechanisms and genetic control. ${ }^{12}$ In addition, retrospective meta-analyses sometimes only include focused or hypothesis-driven studies adopting a region of interest approach (for example, ACC, OFC) with no information on other regions or studies that use coarse or unspecific anatomical regions such as 'frontal lobe'. ${ }^{3-8}$ These approaches may not resolve more subtle or localized patterns of effects.

Here we addressed some of these issues by performing the largest coordinated worldwide meta-analysis of cortical structural abnormalities in patients diagnosed with MDD relative to healthy controls. We extracted cortical thickness and surface area estimates in 2148 MDD patients and 7957 healthy individuals using harmonized data analysis strategies across all sites. ${ }^{13}$ Compared to healthy controls, adult MDD studies generally report cortical thinning, but adolescent MDD studies have reported both cortical thinning and thickening ${ }^{14-17}$ during mid-to-late adolescence. These apparent differences prompted us to analyse adolescent and adult patients separately, with adults defined here as individuals aged $>21$ years. We set the age cut-off for adult versus adolescent analyses at $\leqslant 21$ years, based on 1) evidence of accelerated cortical thinning followed by decelerated thinning in young adulthood during normal brain development ${ }^{18}$ and 2) the presence of a positive correlation between depressive symptoms and ventromedial PFC in individuals with MDD up to 22 years old. ${ }^{19}$ Additional stratifying variables were single vs recurrent episodes, antidepressant medication use, index episode severity and, in the adult sample, adolescent- vs adult-onset.

\section{MATERIALS AND METHODS}

\section{Samples}

The ENIGMA MDD Working Group currently includes 20 international groups with neuroimaging and clinical data from MDD patients and healthy controls (participating sites are mapped in Supplementary Figure S1). Overall, we analysed data from 10105 people, including 2148 MDD patients and 7957 healthy controls. Each sample's demographics are detailed in Supplementary Table S1 and clinical characteristics in Supplementary Table S2. Supplementary Table S3 lists exclusion criteria for study enrolment. All participating sites obtained approval from local institutional review boards and ethics committees, and all study participants provided written informed consent.

\section{Image processing and analysis}

Structural T1-weighted MRI brain scans were acquired at each site and analysed locally using harmonized analysis and quality-control protocols from the ENIGMA consortium; in this case, all cortical parcellations were performed with the freely available and validated segmentation software FreeSurfer (versions 5.1 and 5.3). ${ }^{20}$ Image acquisition parameters and software descriptions are given in Supplementary Table S4. Segmentations of 68 (34 left and 34 right) cortical gray matter regions based on the Desikan-Killiany atlas ${ }^{21}$ and two whole-hemisphere measures were visually inspected and statistically evaluated for outliers following standardized ENIGMA protocols (http://enigma.ini.usc.edu/protocols/imaging-protocols). Further details on image exclusion criteria and quality control may be found in Supplementary Information SI1.

\section{Statistical framework for meta-analysis}

We examined group differences in cortical thickness and surface area between patients and controls within each sample using multiple linear regression models. In the primary analysis, the outcome measures were from each of 70 cortical regions of interest (68 regions and two wholehemisphere average thickness or total surface area measures). A binary indicator of diagnosis $(0=$ controls, $1=$ patients $)$ was the predictor of interest. All models were adjusted for age and sex. Additional covariates were included whenever necessary to control for scanner differences within each sample. To ease comparisons with prior work, ${ }^{2,22}$ effect size estimates were calculated using Cohen's $d$ metric computed from the $t$-statistic of the diagnosis indicator variable from the regression models. Similarly, for models testing interactions (that is, sex-by-diagnosis and 


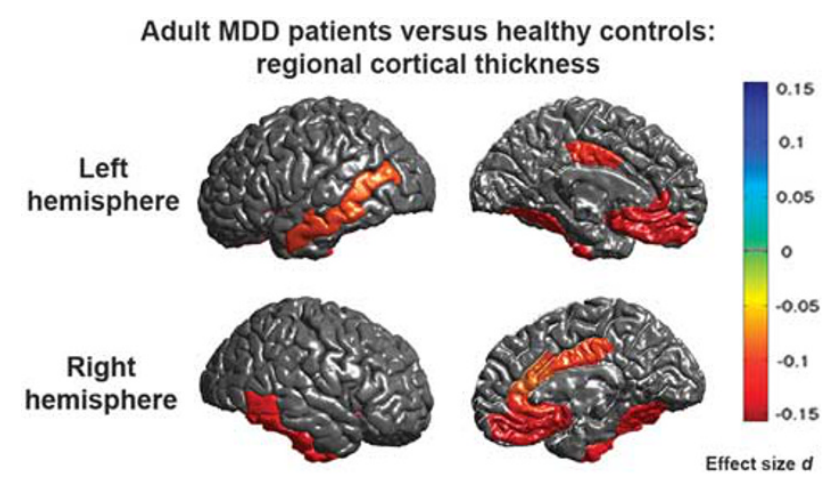

Figure 1. Meta-analysis effect sizes for regions with a significant $\left(P_{\mathrm{FDR}}<0.05\right)$ cortical thinning in adult major depressive disorder (MDD) patients compared to healthy controls. Negative effect sizes $d$ indicate cortical thinning in MDD compared to controls.

age-by-diagnosis) a multiplicative predictor was the predictor of interest with the main effect of each predictor included in the model and the effect size was calculated using the same procedure.

To detect potentially different effects of major depression with age, we separately analysed adolescent (age $\leqslant 21$ years) and adult participants ( $>21$ years). Within the adolescent and adult divisions, we tested stratified models that split patients based on stage of illness (first episode vs recurrent). Furthermore, we examined associations between symptom severity at the time of scanning using the 17-item Hamilton Depression Rating Scale (HDRS-17) $)^{23}$ and the Beck Depression Inventory (BDI-II) ${ }^{24}$ and cortical thickness and surface area. Within the adult division, we stratified patients based on age at illness onset (adolescent-onset $\leqslant 21$ years; adultonset $>21$ years ${ }^{25}$ ). Results of models that split patients based on antidepressant use at the time of their scan are reported in Supplementary Information SI1. Included samples and total sample sizes for each model are listed in the tables in 'Results' section. Throughout the manuscript, we report $P$-values corrected for multiple comparisons using the BenjaminiHochberg procedure ${ }^{26}$ to ensure a false-discovery rate (FDR) limited at $5 \%$ for 70 measures (34 left hemisphere regions, 34 right hemisphere regions and 2 full-hemisphere measures, for left and right).

All regression models and effects size estimates were computed at each site separately and a final Cohen's $d$ effect size estimate was obtained using an inverse variance-weighted random-effect meta-analysis model in $\mathrm{R}$ (metafor package, version 1.9-118). Only for the meta-analyses on correlation with symptom severity scores and number of episodes in recurrent patients, predictors were treated as continuous variables, so effect sizes were expressed as partial-correlation Pearson's $r$ after removing nuisance variables (age, sex, and scan site). The final meta-analysed partialcorrelation $r$ was estimated with the same inverse variance-weighted random-effect meta-analysis model. See Supplementary Information SI1 for full meta-analysis details.

\section{Moderator analyses with meta-regression}

The methods and results of the moderator analyses, using meta-regression analyses to test whether individual site characteristics explained a significant proportion of the variance in effect sizes across sites in the meta-analyses, are reported in Supplementary Information SI1.

\section{RESULTS}

Adults

Cortical thickness and surface area differences between MDD patients and controls. We found significant and consistent thinner cortices in the frontal and temporal lobes of adult depressed patients $(N=1902)$ compared to controls $(N=7658)$ in the bilateral medial OFC, fusiform gyrus, insula, rostral anterior and posterior cingulate cortex and unilaterally in the left middle temporal gyrus, right inferior temporal gyrus and right caudal ACC (see Figure 1 for significant regions, Supplementary Figure S11 for forest plots and Table 1 for full cortical thickness effects). Regions are listed in all tables in order of effect size, from the strongest to the weakest effect size. None of the regions analysed showed significant differences in cortical surface area (Supplementary Table S19) or evidence of sex-by-diagnosis or age-by-diagnosis interaction effects (Supplementary Tables S5, S6, S20 and S21).

First vs recurrent episode adult $M D D$. Adult patients with recurrent depression $(N=1302)$ compared to controls $(N=7450)$ revealed cortical thinning in left medial OFC (Supplementary Figures S2 and S12). First-episode patients $(N=535)$ compared to controls $(N=7253)$ showed more widespread cortical thinning in bilateral fusiform gyrus, rostral ACC and insula and left medial orbitofrontal and superior frontal cortex, right caudal anterior and posterior cingulate cortex and right isthmus cingulate cortex (Supplementary Figures S3 and S13). No differences were detected between recurrent and first-episode patients (Supplementary Table S9). Similar to the overall MDD group analysis, no cortical surface area differences were detected (Supplementary Tables S22-S24), and we found no significant correlations between thickness and surface area and the number of depressive episodes in recurrent patients $(N=496$; Supplementary Table S10).

Age of onset in adult MDD. Cortical thinning was observed in patients with an adult age of illness onset ( $>21$ years, $N=1214$ ) compared to controls $(N=3329)$ in bilateral insula, rostral anterior, posterior and isthmus cingulate cortex, fusiform gyrus, medial OFC, right caudal ACC and right inferior temporal gyrus (Supplementary Figures S4 and S14). We did not detect significant differences in cortical thickness in patients with an adolescent age of onset ( $\leqslant 21$ years, $N=472)$, compared to controls $(N=2885)$, (Supplementary Table S12) and when comparing adolescentonset and adult-onset patients directly (Supplementary Table S13). Similarly, no surface area differences were detected in these subgroup analyses (Supplementary Tables S26-S28).

Correlation with symptom severity in adult patients. None of the cortical thickness measurements were correlated with symptom severity at study inclusion using the HDRS-17 $(N=776)$ and BDI-II $(N=943)$ questionnaires (Supplementary Tables S17 and S18). For surface area measurements, no associations were found with the HDRS-17 (Supplementary Table S32) and weak negative correlations were detected for BDI-II scores and surface area of the bilateral precuneus, left frontal pole and left postcentral gyrus (Supplementary Table S33, Supplementary Figures S7 and S17).

\section{Adolescents}

Cortical thickness and surface area differences between adolescent MDD patients and controls. Left and right hemisphere total surface area was smaller in depressed adolescent patients $(N=213)$ compared to adolescent controls $(N=294)$. Regionally, surface area reductions were observed in bilateral lingual gyrus and pericalcarine gyrus, left lateral occipital cortex, left medial OFC, left precentral gyrus, right inferior parietal cortex, right superior frontal gyrus and right postcentral gyrus (see Figure 2 and Supplementary Figure S18 and Table 2 for full tabulation of effects). No cortical thickness differences were detected between adolescent MDD patients and controls (Supplementary Table S34). Further, no cortical regions showed age-by-diagnosis or sex-by-diagnosis interaction effects (Supplementary Tables S35, S36, S45 and S46).

First vs recurrent episode adolescent MDD. Adolescents with recurrent depression $(N=104)$ showed reductions in left and right hemisphere overall surface area compared to controls $(N=142)$. Regionally, surface area reductions were observed in bilateral inferior parietal cortex and caudal middle frontal gyrus and left fusiform gyrus, left lateral occipital cortex, left precuneus, left 
Table 1. Full meta-analytic results for thickness of each structure for adult MDD patients vs controls comparison controlling for age, sex and scan center

\begin{tabular}{|c|c|c|c|c|c|c|c|c|c|}
\hline & Cohen's $\mathrm{d}^{\mathrm{a}}$ (MDD vs CTL) & s.e. & $95 \% \mathrm{Cl}$ & \% Difference & P-value & FDR P-value & $1^{2}$ & No. of controls & No. of patients \\
\hline Left medial orbitofrontal cortex & -0.134 & 0.038 & $(-0.208$ to -0.059$)$ & -1.035 & 4.35E-04 & 0.015 & 23.025 & 7609 & 1888 \\
\hline Right medial orbitofrontal cortex & -0.131 & 0.047 & $(-0.224$ to -0.039$)$ & -1.102 & 0.006 & 0.035 & 46.981 & 7628 & 1896 \\
\hline Left rostral anterior cingulate cortex & -0.130 & 0.044 & $(-0.216$ to -0.044$)$ & -1.264 & 0.003 & 0.030 & 39.185 & 7656 & 1896 \\
\hline Right lateral orbitofrontal cortex & -0.120 & 0.048 & $(-0.214$ to -0.026$)$ & -0.704 & 0.012 & 0.058 & 48.548 & 7644 & 1902 \\
\hline Left fusiform gyrus & -0.117 & 0.030 & $(-0.176$ to -0.058$)$ & -0.576 & $9.51 \mathrm{E}-05$ & 0.007 & $<0.001$ & 7645 & 1896 \\
\hline Right inferior temporal gyrus & -0.117 & 0.036 & $(-0.188$ to -0.046$)$ & -0.633 & $1.29 \mathrm{E}-03$ & 0.018 & 18.006 & 7640 & 1885 \\
\hline Right fusiform gyrus & -0.116 & 0.042 & ( -0.198 to -0.033$)$ & -0.564 & 0.006 & 3.47E-02 & 34.668 & 7649 & 1898 \\
\hline Right insula & -0.115 & 0.041 & $(-0.195$ to -0.035$)$ & -0.624 & 0.005 & 0.035 & 31.271 & 7651 & 1895 \\
\hline Left insula & -0.111 & 0.034 & $(-0.177$ to -0.045$)$ & -0.582 & $9.34 \mathrm{E}-04$ & 0.018 & 10.683 & 7652 & 1898 \\
\hline Left isthmus cingulate cortex & -0.104 & 0.046 & $(-0.194$ to -0.014$)$ & -0.797 & 0.024 & 0.100 & 44.546 & 7655 & 1897 \\
\hline Left posterior cingulate cortex & -0.099 & 0.030 & $(-0.158$ to -0.04$)$ & -0.618 & 0.001 & 0.018 & 0.030 & 7654 & 1900 \\
\hline Right rostral anterior cingulate cortex & -0.098 & 0.034 & $(-0.165$ to -0.031$)$ & -0.993 & 0.004 & 0.034 & 12.104 & 7651 & 1899 \\
\hline Right posterior cingulate cortex & -0.093 & 0.030 & $(-0.152$ to -0.034$)$ & -0.624 & 0.002 & 0.022 & 0.028 & 7654 & 1900 \\
\hline Left middle temporal gyrus & -0.090 & 0.031 & $(-0.151$ to -0.028$)$ & -0.531 & 0.004 & 0.034 & 2.685 & 7591 & 1822 \\
\hline Right middle temporal gyrus & -0.088 & 0.035 & $(-0.156$ to -0.021$)$ & -0.492 & 0.011 & 0.053 & 13.072 & 7639 & 1886 \\
\hline Right caudal anterior cingulate cortex & -0.080 & 0.030 & $(-0.139$ to -0.021$)$ & -0.892 & 0.008 & 0.041 & $<0.001$ & 7655 & 1898 \\
\hline Right superior frontal gyrus & -0.078 & 0.034 & $(-0.145$ to -0.011$)$ & -0.379 & 0.023 & 0.099 & 12.509 & 7649 & 1900 \\
\hline Right banks superior temporal sulcus & -0.074 & 0.034 & $(-0.14$ to -0.008$)$ & -0.528 & 0.028 & 0.103 & 9.841 & 7613 & 1827 \\
\hline Left pars orbitalis & -0.073 & 0.044 & $(-0.158$ to 0.012$)$ & -0.533 & 0.094 & 0.212 & 38.655 & 7653 & 1900 \\
\hline Left parahippocampal gyrus & -0.072 & 0.033 & $(-0.137$ to -0.007$)$ & -0.895 & 0.030 & 0.103 & 8.631 & 7648 & 1896 \\
\hline Right isthmus cingulate cortex & -0.071 & 0.038 & $(-0.146$ to 0.004$)$ & -0.563 & 0.065 & 0.174 & 24.975 & 7651 & 1897 \\
\hline Right pars orbitalis & -0.070 & 0.042 & $(-0.152$ to 0.013$)$ & -0.501 & 0.100 & 0.212 & 35.595 & 7655 & 1900 \\
\hline Left superior frontal gyrus & -0.066 & 0.030 & $(-0.125$ to -0.008$)$ & -0.336 & 0.027 & 0.103 & $<0.001$ & 7652 & 1899 \\
\hline Left inferior parietal cortex & -0.063 & 0.044 & $(-0.149$ to 0.022$)$ & -0.359 & 0.147 & 0.277 & 38.619 & 7638 & 1894 \\
\hline Left pars opercularis & -0.063 & 0.030 & $(-0.122$ to -0.004$)$ & -0.299 & 0.037 & 0.123 & 0.003 & 7655 & 1897 \\
\hline Right frontal pole & -0.062 & 0.036 & $(-0.133$ to 0.009$)$ & -0.650 & 0.085 & 0.204 & 18.189 & 7657 & 1899 \\
\hline Right parahippocampal gyrus & -0.061 & 0.030 & $(-0.12$ to -0.002$)$ & -0.665 & 0.042 & 0.134 & 0.010 & 7654 & 1897 \\
\hline Left banks superior temporal sulcus & -0.058 & 0.031 & $(-0.118$ to 0.002$)$ & -0.423 & 0.059 & 0.173 & $<0.001$ & 7571 & 1781 \\
\hline Left hemisphere average thickness & -0.057 & 0.031 & ( -0.117 to 0.003$)$ & -0.209 & 0.065 & 0.174 & 2.346 & 7658 & 1902 \\
\hline Right entorhinal cortex & -0.055 & 0.030 & ( -0.115 to 0.004$)$ & -0.657 & 0.068 & 0.177 & $<0.001$ & 7602 & 1862 \\
\hline Left pars triangularis & -0.054 & 0.030 & $(-0.112$ to 0.005$)$ & -0.326 & 0.074 & 0.185 & $<0.001$ & 7651 & 1897 \\
\hline Right supramarginal gyrus & -0.053 & 0.044 & ( -0.139 to 0.032$)$ & -0.273 & 0.223 & 0.370 & 38.371 & 7633 & 1874 \\
\hline Right transverse temporal gyrus & -0.051 & 0.030 & $(-0.11$ to 0.008$)$ & -0.413 & 0.088 & 0.204 & $<0.001$ & 7622 & 1894 \\
\hline Left inferior temporal gyrus & -0.049 & 0.035 & $(-0.117$ to 0.019$)$ & -0.273 & 0.158 & 0.291 & 12.727 & 7630 & 1872 \\
\hline Right hemisphere average thickness & -0.049 & 0.033 & $(-0.113$ to 0.015$)$ & -0.179 & 0.135 & 0.263 & 8.061 & 7658 & 1902 \\
\hline Left lateral orbitofrontal cortex & -0.046 & 0.031 & ( -0.107 to 0.014$)$ & -0.280 & 0.130 & 0.260 & 1.851 & 7638 & 1898 \\
\hline Left supramarginal gyrus & -0.045 & 0.037 & $(-0.118$ to 0.027$)$ & -0.244 & 0.220 & 0.370 & 19.395 & 7609 & 1864 \\
\hline Left caudal anterior cingulate cortex & -0.042 & 0.036 & ( -0.113 to 0.028$)$ & -0.481 & 0.240 & 0.377 & 17.908 & 7650 & 1900 \\
\hline Right inferior parietal cortex & -0.041 & 0.044 & ( -0.127 to 0.044$)$ & -0.235 & 0.343 & 0.501 & 39.065 & 7641 & 1897 \\
\hline Left entorhinal cortex & -0.041 & 0.038 & $(-0.115$ to 0.033$)$ & -0.471 & 0.276 & 0.420 & 21.951 & 7605 & 1866 \\
\hline Right rostral middle frontal gyrus & -0.038 & 0.045 & $(-0.127$ to 0.051$)$ & -0.183 & 0.401 & 0.561 & 42.738 & 7650 & 1899 \\
\hline Left rostral middle frontal gyrus & -0.037 & 0.030 & (-0.096 to 0.022$)$ & -0.178 & 0.224 & 0.370 & 0.467 & 7653 & 1899 \\
\hline Left transverse temporal gyrus & -0.035 & 0.030 & $(-0.094$ to 0.024$)$ & -0.277 & 0.243 & 0.377 & $<0.001$ & 7635 & 1895 \\
\hline Right pars triangularis & -0.031 & 0.046 & ( -0.122 to 0.059$)$ & -0.179 & 0.501 & 0.674 & 44.727 & 7645 & 1897 \\
\hline Right superior temporal gyrus & -0.031 & 0.030 & $(-0.09$ to 0.029$)$ & -0.184 & 0.314 & 0.468 & $<0.001$ & 7587 & 1820 \\
\hline Left precuneus & -0.024 & 0.039 & $(-0.101$ to 0.053$)$ & -0.115 & 0.541 & 0.701 & 27.766 & 7649 & 1893 \\
\hline Left lateral occipital cortex & -0.023 & 0.044 & ( -0.109 to 0.063$)$ & -0.131 & 0.605 & 0.756 & 39.881 & 7645 & 1898 \\
\hline Right precentral gyrus & -0.022 & 0.040 & $(-0.101$ to 0.057$)$ & -0.132 & 0.581 & 0.739 & 29.869 & 7643 & 1894 \\
\hline Left precentral gyrus & -0.020 & 0.031 & $(-0.08$ to 0.04$)$ & -0.115 & 0.516 & 0.681 & 2.091 & 7637 & 1895 \\
\hline Right pars opercularis & -0.017 & 0.044 & ( -0.103 to 0.069$)$ & -0.087 & 0.694 & 0.823 & 39.291 & 7651 & 1896 \\
\hline Left caudal middle frontal gyrus & -0.014 & 0.041 & ( -0.094 to 0.067$)$ & -0.069 & 0.741 & 0.844 & 32.111 & 7647 & 1898 \\
\hline Right lingual gyrus & -0.012 & 0.030 & $(-0.071$ to 0.047$)$ & -0.069 & 0.692 & 0.823 & $<0.001$ & 7641 & 1894 \\
\hline
\end{tabular}




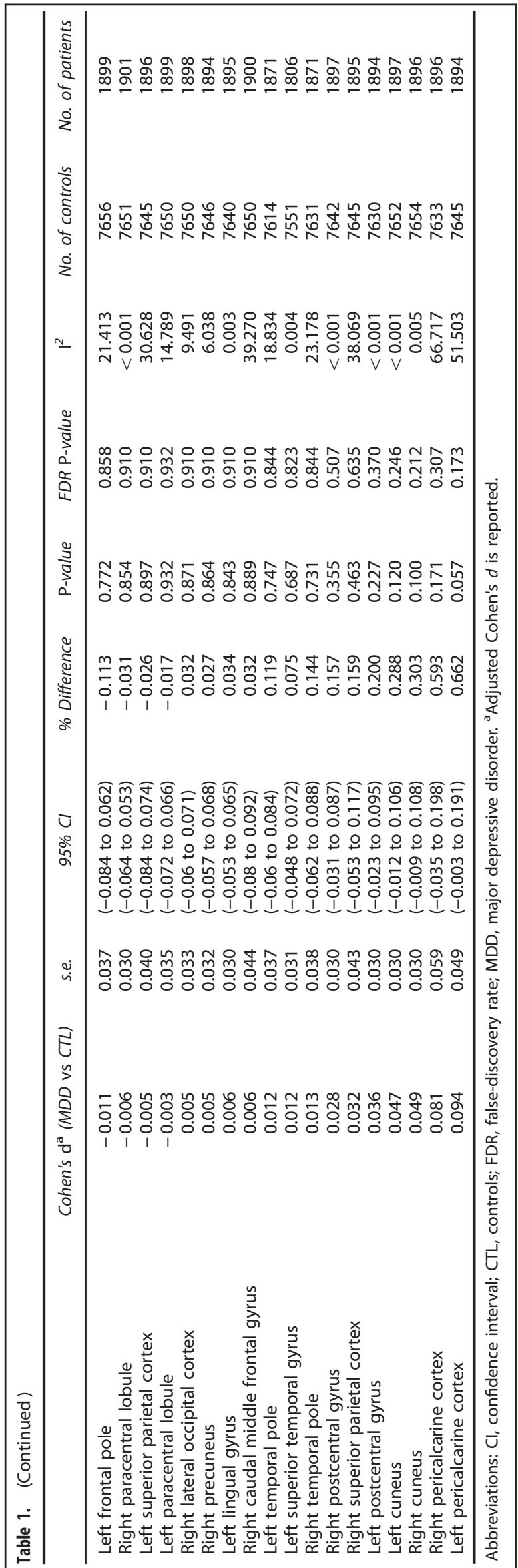

Adolescent MDD versus healthy controls: regional cortical surface area

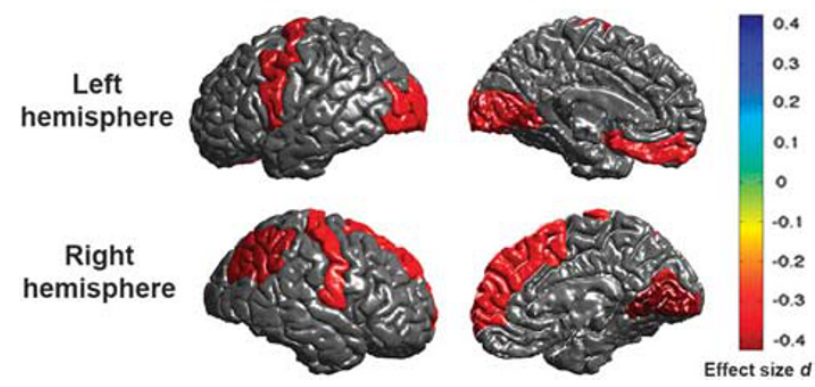

Figure 2. Meta-analysed effect sizes for regions with a significant $\left(P_{\mathrm{FDR}}<0.05\right)$ decrease in cortical surface area in adolescent major depressive disorder (MDD) patients compared to healthy controls. Negative effect sizes $d$ indicate lower cortical surface area in MDD compared to controls.

superior parietal cortex, left medial OFC, right banks of the superior temporal sulcus, right lingual gyrus, right pericalcarine gyrus and right postcentral gyrus (Supplementary Figures S8 and S19, Supplementary Table S48). First-episode patients $(N=80)$ showed no detectable differences, when compared to controls $(N=154)$ or the recurrent adolescent MDD group (Supplementary Tables S47 and S49). No cortical thickness differences were found in adolescent MDD for first-episode or recurrence subgroups (Supplementary Tables S37-S39); similarly, no correlations with the number of episodes were detected for surface area or thickness in recurrent adolescent MDD patients (Supplementary Tables S40 and S50).

Correlations with symptom severity in adolescent MDD. We did not detect significant differences in cortical thickness or surface area when examining the effects of symptom severity at study inclusion using the HDRS-17 $(N=134)$ questionnaire (Supplementary Tables S44 and S54), whereas BDI-II scores were available only for a small group of adolescent patients $(N=31)$, precluding meaningful comparisons.

Moderating effects on cortical thickness and surface area Results of the moderator analyses can be found in the Supplementary Information.

\section{DISCUSSION}

In the largest analysis to date of cortical structural measures, we applied an individual participant data-based meta-analytic approach to brain MRI data from $>10000$ people, of whom around one-fifth were affected by MDD. We found significant differences in cortical brain structures in adolescent and adult MDD and specific associations with clinical characteristics.

Cortical thickness

Adult MDD patients had cortical thickness deficits in 13 (of 68) regions examined. Cortical thinning was generally observed bilaterally, in regions that encompassed the medial PFC, rostral anterior and posterior cingulate cortex, insula and fusiform gyrus. Unilateral effects were observed in left middle temporal gyrus and right inferior temporal and right caudal ACC. Our findings of lower cortical thickness in medial PFC and ACC are consistent with prior meta-analyses. ${ }^{3-8}$ Our findings extend previous findings by demonstrating structural abnormalities in the temporal lobe (middle and inferior temporal and fusiform gyri), posterior cingulate cortex and insula. The large sample also adds to our 
Table 2. Full meta-analytic results for surface area of each structure for adolescent MDD patients vs controls comparison controlling for age, sex and scan center

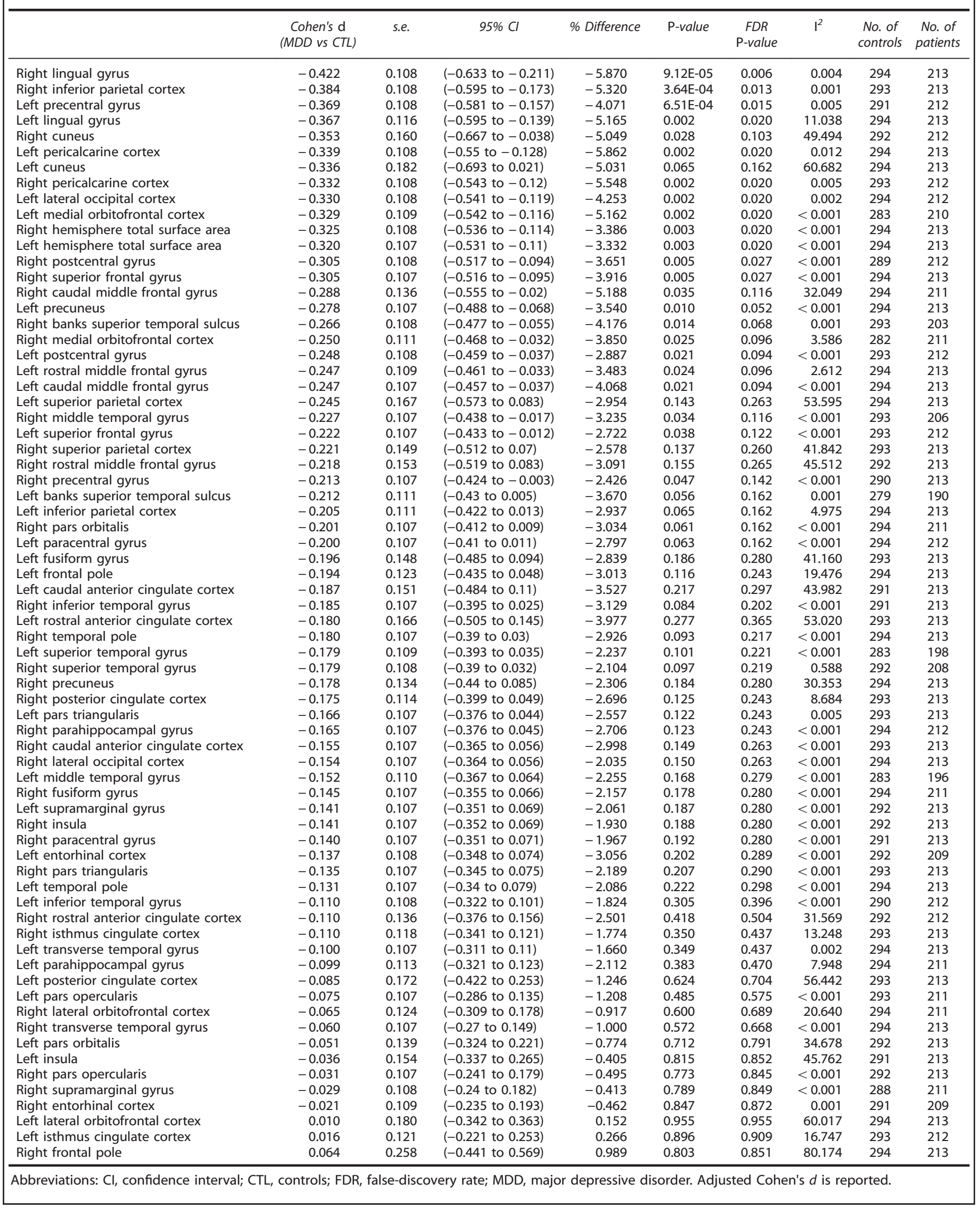


understanding of how reproducible and consistent these effects are likely to be when surveying cohorts worldwide.

A key feature of these regions is their close interaction with the limbic system, consistent with the general pathophysiological model of MDD that posits dysfunctional limbic-cortical circuits. $^{27,28}$ The dorsal and rostral ACC are functionally heterogeneous, supporting task monitoring, conflict detection, emotion regulation, social cognition and executive functions. ${ }^{29}$ The insular cortex is similarly multifunctional and engaged in visceroception, autonomic response regulation and attentional switches (for example, Menon and $\operatorname{Uddin}^{30}$ ). These regions show consistent structural differences in this cross-sectional morphometric study that may contribute to the broad spectrum of emotional, cognitive and behavioural disturbances observed in MDD.

Although effect sizes were relatively small $(d-0.08$ to -0.13 , percentage of difference $-0.5 \%$ to $-1.3 \%$, with overall low-tomedium heterogeneity among studies; that is, $l^{2}$ for most regions between $0 \%$ and $50 \%$ ) and in the range of previously reported hippocampal volume reduction, ${ }^{2}$ the medial OFC showed the largest effect sizes ( $d-0.13$, percentage of difference $-1.1 \%)$. The lower medial wall of the PFC (medial OFC according to the Desikan-Killiany atlas ${ }^{21}$ in FreeSurfer) contains the subgenual ACC (sgACC), subcallosal gyrus and medial OFC and has dense connections to the hypothalamus as the primary site of stress response regulation. ${ }^{31}$ These findings concur with postmortem findings of OFC structural deficits, ${ }^{32}$ OFC/sgACC-specific volumetric meta-analyses, ${ }^{8,33}$ correlations between OFC thickness and cortisol levels $s^{34}$ and evidence of functional derangement of the sgACC in depression. ${ }^{35}$ Recently, right medial OFC thickness measured at baseline in healthy adolescent girls proved a strong predictor of the onset of depression in a multivariate model. ${ }^{36}$ The ventromedial PFC and OFC (including the sgACC) are critically involved in reinforcement learning, ${ }^{37}$ fear responsiveness and the adaptive control of emotions, ${ }^{38}$ which are disturbed in MDD, and have been associated with both a non-response to therapy ${ }^{39,40}$ and a more unfavourable course of the illness. ${ }^{41}$ Distinct from our hippocampal volume finding, ${ }^{2}$ these effects were detectable already in first-episode patients with a medial OFC/ACC and insular focus, indifferent from recurrent patients who showed less widespread changes compared to controls. Further, no correlations with the number of episodes and no age-by-diagnosis effects were detected. Although these observations are based on cross-sectional data, we add to limited and conflicting reports of longitudinal volumetric changes in MDD $^{42,43}$ which suggest that progressive cortical abnormalities with growing disease load does not appear to be a general feature of depression.

With regard to age at onset, no significant differences were found between adult patients with an adolescent-onset $(\leqslant 21$ years) and controls. In contrast, adult-onset was associated with significant cortical thinning in numerous frontal, cingulate and temporal regions. Interestingly, our prior work ${ }^{2}$ showed hippocampal volume alterations in adolescent-onset but not adultonset patients. This result may suggest differential effects of stress-related remodelling or interactions with brain maturational mechanisms at different periods of disease onset. Cortical structural deficits were not found in adolescent-onset adult patients. This, however, may in part be due to lower statistical power in the smaller adolescent-onset compared with the adultonset patient samples ( $N=472$ vs $N=1214$ ). In addition, the lack of effects could perhaps be explained by the fact that adolescentonset patients were younger than the adult-onset groups. Hence, greater cortical thinning in MDD may be more pronounced in adult-onset patients if the disease effects interact with increased aging of the brain, ${ }^{40,44}$ but see also Truong et al. ${ }^{9}$ Following this logic, we performed a post-hoc moderator analysis examining the effects of mean age of patients in each sample on cortical thickness differences between adolescent-onset (adult) patients and controls. Samples with a higher mean age of patients indeed showed greater cortical thinning in the adolescent-onset group compared with controls (Supplementary Figure S22). Though not robust to conservative correction for multiple comparisons (trendlevel $P_{\mathrm{FDR}}=0.09$ for the left medial OFC), this pattern fits the lack of detected thickness differences in our adolescent MDD vs adolescent controls analysis. Prior studies have reported mixed results with regard to cortical abnormalities in adolescent MDD, showing increased, ${ }^{15,17}$ decreased ${ }^{14-16}$ or no differences in cortical thickness. ${ }^{45}$ Cortical thickness decreases linearly during adolescence $^{46-49}$ owing to synaptic pruning, myelination and other remodelling effects. ${ }^{50}$ In adolescent MDD, anxious and depressed symptoms have been associated with greater cortical thickness. ${ }^{19}$ In contrast, our current results and prior reports ${ }^{3-8}$ provide consistent evidence for cortical thinning in adult MDD. These opposite effects would suggest a delay in maturation (that is, delay in thinning) of cortical thickness in adolescent MDD, resulting in greater cortical thickness during various stages of brain maturation but thinner cortex eventually. A possible explanation for the lack of cortical thickness effects in the current study is that $70 \%$ of our adolescents were 18-21 years, perhaps older than the most sensitive period to detect maturation delays. ${ }^{17,19}$ Of note, although not significant, the left lateral OFC showed a medium effect size $(d-0.31$, percentage of difference $-1.9 \%)$ for cortical thinning in adolescent MDD compared to controls, whereas cortical alterations in other regions were less clear.

\section{Cortical surface area}

Adult MDD patients showed no surface area abnormalities compared to controls. However, adolescent patients revealed smaller left and right hemisphere total surface areas, reflecting a diffuse pattern of local surface area deficits (effect sizes $d$ between -0.31 to -0.42 , percentage of difference -3.3 to $-5.9 \%$ ). Similar to cortical thickness alterations in adult MDD we observed surface area deficits in medial OFC and superior frontal gyrus, but also in primary and higher order visual, somatosensory and motor areas. These deficits were observed in recurrent patients, suggesting a negative effect of multiple episodes.

Cortical thinning starts from 2 to 4 years of age and continues across the lifespan, but overall cortical surface area follows a nonlinear and nonmonotonic developmental trajectory. The cortical surface expands until about 12 years, remains relatively stable and then decreases with age. ${ }^{46-49}$ Development of cortical thickness and surface area are genetically independent ${ }^{12}$ and result from different neurobiological processes, ${ }^{50}$ representing distinct features of cortical development and aging. Cortical surface area abnormalities were not detected in our early-onset adult MDD patients, despite greater statistical power than for the adolescent analyses, so smaller cortical surface area in adolescent MDD may indicate delayed cortical maturation (that is, delayed expansion). Some regions with surface area abnormalities, including medial occipital regions (lingual gyrus), inferior parietal cortex, precentral gyrus, medial OFC and superior frontal gyrus, mature over a more prolonged time course during adolescence $^{47,49}$ and may be especially prone to a delay in maturation in adolescent MDD. Such delayed maturation may alter functional connections with other regions through decreases in growth and branching of dendritic trees and the number of synapses associated with gray matter volume, ${ }^{51}$ which may persist into adult MDD even if surface area measures normalize when transitioning into adulthood. The absence of cortical surface area abnormalities in the adult MDD patients with an early age of onset of depression could indicate such normalization; importantly, however, we still detected weak negative associations between severity of depressive symptoms and bilateral precuneus, left frontal pole and left postcentral gyrus surface area. 
To our knowledge, alterations in cortical surface area abnormalities have not been evaluated in adolescents with MDD. Surface area deficits of the ventromedial PFC and precuneus in children and adolescents have been associated with higher anxiety, ${ }^{52}$ of the lingual and temporal gyri in children with childhood maltreatment, ${ }^{53}$ of prefrontal regions in children experiencing early life adversity ${ }^{54}$ and of the OFC in adolescents with conduct disorder. ${ }^{55}$ Importantly, early life stress, symptoms of anxiety and externalizing problems in childhood and early adolescence are all risk factors for early-onset MDD. ${ }^{56,57}$

Cortical thickness and surface area abnormalities were mainly observed in first-episode MDD and adolescent MDD, respectively; this may indicate that cortical alterations are a feature of more heterogeneous MDD samples, including adolescent and firstepisode adult MDD individuals who may go on to other outcomes, including bipolar or psychotic disorders, instead of adult MDD samples with a more 'pure' depressive phenotype (in our study characterized by recurrent MDD and adult MDD with an adolescent-onset of depression in whom the illness is confirmed over time). Indeed, lower surface areas in many of the same regions we observed in adolescent MDD in the current study were prospectively predictive of poor functional outcomes in young people with a clinically defined risk of developing psychosis. ${ }^{58}$ Similar analyses currently underway in the ENIGMA Schizophrenia and ENIGMA Bipolar Disorder working groups may clarify whether regional cortical surface area and thickness are altered to a greater extent in individuals with schizophrenia and bipolar disorder than the alterations we observed in (adult) MDD. Nonetheless, prospective studies are needed to confirm this heterogeneity hypothesis.

\section{Limitations}

We did not adjust the regional comparisons for average thickness or total surface, respectively, as our main question was directed towards regional MDD-related changes instead of identifying regional effects that exceed a global effect. In contrast to surface area measures, which are highly associated with global measures of the brain (for example, intracranial volume, as a proxy for overall brain size), cortical thickness does not scale proportionately with brain size. ${ }^{59}$ In the current study, global deficits in cortical surface area (indicated by smaller left and right total surface area) were observed in adolescents with MDD. Therefore, our surface area results need to be interpreted as a diffuse, global surface deficit in adolescent MDD, with potential additional regional accentuation.

Furthermore, we used a $\leqslant 21$-year cutoff for adolescent vs adult MDD (cf. 'Introduction' section) consistent with our previous work. $^{2}$ Definitions of adolescent MDD in the literature are not consistent, so alternative definitions might yield different results. Ideally, age and age of onset effects on brain abnormalities in MDD should be examined using a dimensional approach. However, in the current meta-analysis the statistical analyses were performed within each site, precluding this approach as few samples covered the entire lifespan. In addition, the age distribution of the adolescents ( $9 \%$ between 12 and 16 years, $21 \%$ between 16 and 18 years, $70 \% \geqslant 18$ years) and the limited adolescent sample size (while larger than prior reports) may not be ideally sensitive to detect age-by-diagnosis interaction and cortical thickness effects. Future addition of more adolescent MDD samples to reflect a balanced age distribution may aid in detecting cortical changes associated with MDD at different stages of brain development.

In addition, when combining already collected data across worldwide samples, data collection protocols are not prospectively harmonized. Imaging acquisition protocols and clinical assessments therefore differed across studies, which limits analysis of sources of heterogeneity. The current study did not allow a reliable investigation of antidepressant medication effects on cortical structure because of its cross-sectional design and lack of detailed information on history, duration and type and dosage of antidepressant treatment. Still, in Supplementary Information SI1 we report on comparisons between patients taking antidepressant, antidepressant-free patients and controls. Adult patients using antidepressants showed robust and widespread effects of cortical thinning, whereas non-users showed cortical thinning only in the left medial OFC. However, this cross-sectional finding should not be interpreted as contradicting generally observed neuroprotective effects of antidepressants. ${ }^{60}$ It is likely confounded by clinical standards recommending antidepressant use mainly for severe or chronic MDD. In adolescent MDD patients, surface area deficits were observed in antidepressant-free patients and not in adolescents taking antidepressants. Clearly, intervention studies with preantidepressant and postantidepressant treatment comparisons of antidepressants are required to draw valid conclusions on the impact of antidepressant use on cortical structure.

\section{CONCLUSIONS}

Cortical structure is abnormal in numerous brain regions in adult and adolescent MDD. Medial OFC was consistently implicated across analyses -in adults, adolescents and analyses of clinical correlations. This finding reinforces the hypothesized prominent role of this region in depression throughout life. Other than subcortical volumetric effects, cortical thickness changes were robustly detectable in adult patients at their first episode. MDD may dynamically impact cortical development, and vice versa, with different patterns of alterations at different stages of life. Cortical thickness measurements showed greater differences than surface area measures in adult MDD, but consistent surface area deficits were found in adolescent MDD. Cortical thickness and surface area represent distinct morphometric features of the cortex and may be differentially affected by depression at various stages of life. Future (longitudinal) studies are needed to examine dynamic changes in the cortical regions we examined here and to relate such changes to symptom profiles, outcomes and treatment responses in MDD.

\section{CONFLICT OF INTEREST}

The authors declare no conflict of interest. Jair Soares has participated in research funded by Forest, Merck, BMS, GSK and has been a speaker for Pfizer and Abbott. Andrew Mclntosh has received support from Lilly, Janssen, Pfizer and Saccade Diagnostics. Carsten Konrad received fees for an educational program from Aristo Pharma, Janssen-Cilag, Lilly, MagVenture, Servier, and Trommsdorff as well as travel support and speakers honoraria from Janssen, Lundbeck and Servier. Theo G.M. van Erp has consulted for Roche Pharmaceuticals Ltd., and has a contract with Otsuka Phamaceutical Co., Ltd (OPCJ). Knut Schnell has consulted for Roche Pharmaceuticals and Servier Pharmaceuticals. Henrik Walter has received a speaker honorarium from Servier, 9987.

\section{ACKNOWLEDGMENTS}

The ENIGMA-Major Depressive Disorder working group gratefully acknowledges support from the NIH Big Data to Knowledge (BD2K) award (U54 EB020403 to Paul Thompson).

NESDA: The infrastructure for the NESDA study (www.nesda.nl) is funded through the Geestkracht program of the Netherlands Organisation for Health Research and Development (Zon-Mw, grant number 10-000-1002) and is supported by participating universities (VU University Medical Center, GGZ inGeest, Arkin, Leiden University Medical Center, GGZ Rivierduinen, University Medical Center Groningen) and mental health care organizations, see HYPERLINK "http://www.nesda.nl" www.nesda.nl. Lianne Schmaal is supported by The Netherlands Brain Foundation Grant number F2014(1)-24 and the Neuroscience Campus Amsterdam grant (IPB-SE-15-PSYCHSchmaal).

QTIM: QTIM was funded by the Australian National Health and Medical Research Council (Project Grants No. 496682 and 1009064) and US National Institute of Child 
Health and Human Development (RO1HD050735). Baptiste Couvy-Duchesne and Lachlan Strike are supported by a PhD scholarship from the University of Queensland. We are grateful to the twins for their generosity of time and willingness to participate in our study. We also thank the many research assistants, radiographers, and other staff at QIMR Berghofer Medical Research Institute and the Centre for Advanced Imaging, University of Queensland.

MMDP 3T: Ontario Mental Health Foundation.

Bipolar Family Study: The Bipolar Family Study received funding from the European Union's Seventh Framework Programme for research under grant agreement $n^{\circ}$ 602450. This study is also supported by Wellcome Trust award 104036/Z/14/Z.

CODE: The CODE cohort was collected from studies funded by Lundbeck and the German Research Foundation (WA 1539/4-1, SCHN 1204/3-1). Elizabeth Schramm is supported by a Grant of the Deutsche Forschungsgemeinschaft / German Research Association (SCHR 443/11-1).

MPIP: The MPIP Munich Morphometry Sample comprises patients included in Munich Antidepressant Response Signature study and the Recurrent Unipolar Depression (RUD) Case-Control study, and control subjects acquired at the LudwigMaximilians-University, Munich, Department of Psychiatry. We wish to acknowledge Rosa Schirmer, Elke Schreiter, Reinhold Borschke and Ines Eidner for image acquisition and data preparation, and Benno Pütz, Nazanin Karbalai, Darina Czamara, Till Andlauer and Bertram Müller-Myhsok for distributed computing support. We thank Dorothee P. Auer for initiation of the RUD study. The MARS study is supported by a grant of the Exzellenz-Stiftung of the Max Planck Society. This work has also been funded by the Federal Ministry of Education and Research (BMBF) in the framework of the National Genome Research Network (NGFN), FKZ 01 GS0481.

SHIP: The Study of Health in Pomerania (SHIP) is supported by the German Federal Ministry of Education and Research (grants 01ZZ9603, $01 Z Z 0103$ and 01ZZ0403) the Ministry of Cultural Affairs as well as the Social Ministry of the Federal State of Mecklenburg-West Pomerania. MRI scans were supported by Siemens Healthcare, Erlangen, Germany. SHIP-LEGEND was supported by the German Research Foundation (GR1912/5-1).

Rotterdam Study: The Rotterdam Study is supported by the Erasmus MC and Erasmus University Rotterdam; Netherlands Organisation for Scientific Research (NWO); Netherlands Organisation for Health Research and Development (ZonMW); Research Institute for Diseases in the Elderly (RIDE); Netherlands Genomics Initiative; Ministry of Education, Culture and Science; Ministry of Health, Welfare and Sports; European Commission (DG XII); and Municipality of Rotterdam.

Muenster Cohort: The Muenster Neuroimaging Cohort was supported by grants from the German Research Foundation (DFG; grant FOR 2107; DA1151/5-1 to UD) and Innovative Medizinische Forschung (IMF) of the Medical Faculty of Münster (DA120903 to UD, DA111107 to UD, and DA211012 to UD).

Stanford: NIMH Grant R01MH59259 to lan Gotlib, and the National Science Foundation Integrative Graduate Education and Research Traineeship (NSF IGERT) Recipient Award 0801700 and National Science Foundation Graduate Research Fellowship Program (NSF GRFP) DGE-1147470 to Matthew Sacchet.

Melbourne: The study was funded by National Health and Medical Research Council of Australia (NHMRC) Project Grants 1064643 (PI Harrison) and 1024570 (PI Davey).

Houston: Supported in part by NIMH grant R01 085667, The Dunn Foundation, and the Pat Rutherford, Jr. Endowed Chair in Psychiatry to JCS.

Imaging genetics Dublin and Clinical Depression Dublin: The study was supported by a Science Foundation Ireland (SFI) Stokes Professorship Grant to Thomas Frodl.

Novosibirsk: Russian Science Foundation grant \#16-15-00128 to Lyubomir Aftanas. Sydney: This study was supported by the following National Health \& Medical Research Council funding.

sources: Program Grant (No. 566529), Centres of Clinical Research Excellence Grant (No. 264611), Austr.

\section{REFERENCES}

1 World Health Organization. Depression: a global public health concern, 2012. Available at: http://www.who.int/mental_health/management/depression/who paper_depression_wfmh_2012.pdf (accessed 2 October 2015).

2 Schmaal L, Veltman DJ, van Erp TGM, Sämann PG, Frodl T, Jahanshad N et al. Subcortical brain alterations in major depressive disorder: findings from the ENIGMA Major Depressive Disorder Working Group. Mol Psychiatry; e-pub ahead of print 30 June 2015; doi:10.1038/mp.2015.69.

3 Kempton MJ. Structural neuroimaging studies in major depressive disorder. Arch Gen Psychiatry 2011; 68: 675-690.

4 Bora E, Fornito A, Pantelis C, Yücel M. Gray matter abnormalities in major depressive disorder: a meta-analysis of voxel based morphometry studies. J Affect Disord 2012; 138: 9-18.

5 Lai C. Gray matter volume in major depressive disorder: a meta-analysis of voxelbased morphometry studies. Psychiatry Res 2013; 211: 37-46.
6 Zhao $Y-J$, Du M-Y, Huang X-Q, Lui S, Chen Z-Q, Liu J et al. Brain grey matter abnormalities in medication-free patients with major depressive disorder: a meta-analysis. Psychol Med 2014; 44: 2927-2937.

7 Arnone D, Mclntosh AM, Ebmeier KP, Munafò MR, Anderson IM. Magnetic resonance imaging studies in unipolar depression: systematic review and meta-regression analyses. Eur Neuropsychopharmacol 2012; 22: 1-16.

8 Koolschijn PCMP, van Haren NEM, Lensvelt-Mulders GJLM, Hulshoff Pol HE, Kahn RS. Brain volume abnormalities in major depressive disorder: a meta-analysis of magnetic resonance imaging studies. Hum Brain Mapp 2009; 30: 3719-3735.

9 Truong W, Minuzzi L, Soares CN, Frey BN, Evans AC, MacQueen GM et al. Changes in cortical thickness across the lifespan in major depressive disorder. Psychiatry Res 2013; 214: 204-211.

10 Hulvershorn L, Cullen K, Anand A. Toward dysfunctional connectivity: a review of neuroimaging findings in pedriatic major depressive disorder. Brain Imaging Behav 2011; 5: 307-328.

11 Ashburner J, Friston KJ. Voxel-based morphometry-the methods. Neuroimage 2000; 11: 805-821.

12 Winkler AM, Kochunov P, Blangero J, Almasy L, Zilles K, Fox PT et al. Cortical thickness or grey matter volume? The importance of selecting the phenotype for imaging genetics studies. Neuroimage 2010; 53: 1135-1146.

13 Thompson PM, Stein JL, Medland SE, Hibar DP, Vasquez AA, Renteria ME et al. The ENIGMA Consortium: large-scale collaborative analyses of neuroimaging and genetic data. Brain Imaging Behav 2014; 8: 153-182.

14 Boes AD, McCormick LM, Coryell WH, Nopoulos P. Rostral anterior cingulate cortex volume correlates with depressed mood in normal healthy children. Biol Psychiatry 2008; 63: 391-397.

15 Fallucca E, MacMaster FP, Haddad J, Easter P, Dick R, May G et al. Distinguishing between major depressive disorder and obsessive-compulsive disorder in children by measuring regional cortical thickness. Arch Gen Psychiatry 2011; 68: 527-533.

16 Shad MU, Muddasani S, Rao U. Gray matter differences between healthy and depressed adolescents: a voxel-based morphometry study. J Child Adolesc Psychopharmacol 2012; 22: 190-197.

17 Reynolds S, Carrey N, Jaworska N, Langevin LM, Yang X-R, Macmaster FP. Cortical thickness in youth with major depressive disorder. BMC Psychiatry 2014; 14: 83

18 Zhou D, Lebel C, Treit S, Evans A, Beaulieu C. Accelerated longitudinal cortical thinning in adolescence. Neuroimage 2014; 104: 138-145.

19 Ducharme S, Albaugh MD, Hudziak JJ, Botteron KN, Nguyen T-V, Truong C et al. Anxious/depressed symptoms are linked to right ventromedial prefrontal cortical thickness maturation in healthy children and young adults. Cereb Cortex 2014; 24: 2941-2950.

20 Fischl B, Salat DH, Busa E, Albert M, Dieterich M, Haselgrove $C$ et al. Whole brain segmentation: automated labeling of neuroanatomical structures in the human brain. Neuron 2002; 33: 341-355.

21 Desikan RS, Ségonne F, Fischl B, Quinn BT, Dickerson BC, Blacker D et al. An automated labeling system for subdividing the human cerebral cortex on MRI scans into gyral based regions of interest. Neuroimage 2006; 31: 968-980.

22 van Erp TGM, Hibar DP, Rasmussen JM, Glahn DC, Pearlson GD, Andreassen Oa et al. Subcortical brain volume abnormalities in 2028 individuals with schizophrenia and 2540 healthy controls via the ENIGMA consortium. Mol Psychiatry 2016; 21: 547-553.

23 Hamilton M. A rating scale for depression. J Neurol Neurosurg Psychiatry 1960; 23: 56-62.

24 Beck AT, Steer RA, Ball R, Ranieri W. Comparison of Beck Depression Inventories -IA and -II in psychiatric outpatients. J Pers Assess 1996; 67: 588-597.

25 Benazzi F. Classifying mood disorders by age-at-onset instead of polarity. Prog Neuropsychopharmacol Biol Psychiatry 2009; 33: 86-93.

26 Benjamini Y, Hochberg Y. Controlling the false discovery rate: a practical and powerful approach to multiple testing. J R Stat Soc Ser B 1995; 57: 289-300.

27 Mayberg HS. Modulating dysfunctional limbic-cortical circuits in depression: towards development of brain-based algorithms for diagnosis and optimised treatment. Br Med Bull 2003; 65: 193-207.

28 Drevets WC, Price JL, Furey ML. Brain structural and functional abnormalities in mood disorders: implications for neurocircuitry models of depression. Brain Struct Funct 2008; 213: 93-118.

29 Botvinick MM. Conflict monitoring and decision making: reconciling two perspectives on anterior cingulate function. Cogn Affect Behav Neurosci 2007; 7: 356-366

30 Menon V, Uddin LQ. Saliency, switching, attention and control: a network model of insula function. Brain Struct Funct 2010; 214: 655-667.

31 Price JL. Definition of the orbital cortex in relation to specific connections with limbic and visceral structures and other cortical regions. Ann NY Acad Sci 2007; 1121: 54-71. 
32 Rajkowska G, Miguel-Hidalgo J, Wei J, Dilley G, Pittman S, Meltzer H et al. Morphometric evidence for neuronal and glial prefrontal cell pathology in major depression. Biol Psychiatry 1999; 45: 1085-1098.

33 Hajek T, Kozeny J, Kopecek M, Alda M, Höschl C. Reduced subgenual cingulate volumes in mood disorders: a meta-analysis. J Psychiatry Neurosci 2008; 33: 91-99.

34 Liu X, Kakeda S, Watanabe K, Yoshimura R, Abe O, Ide S et al. Relationship between the cortical thickness and serum cortisol levels in drug-naïve, firstepisode patients with major depressive disorder: a surface-based morphometric study. Depress Anxiety 2015; 32: 702-708.

35 Drevets WC, Savitz J, Trimble M. The subgenual anterior cingulate cortex in mood disorders. CNS Spectr 2008; 13: 663-681.

36 Foland-Ross LC, Sacchet MD, Prasad G, Gilbert B, Thompson PM, Gotlib IH. Cortical thickness predicts the first onset of major depression in adolescence. Int $J$ Dev Neurosci 2015; 46: 125-131.

37 Noonan MP, Kolling N, Walton ME, Rushworth MFS. Re-evaluating the role of the orbitofrontal cortex in reward and reinforcement. Eur J Neurosci 2012; 35: 997-1010.

38 Schiller D, Delgado MR. Overlapping neural systems mediating extinction, reversal and regulation of fear. Trends Cogn Sci 2010; 14: 268-276.

39 Redlich R, Opel N, Grotegerd D, Dohm K, Zaremba D, Bürger C et al. The prediction of individual response to electroconvulsive therapy by structural MRI. JAMA Psychiatry 2016 (in press).

40 Mackin RS, Tosun D, Mueller SG, Lee JY, Insel P, Schuff N et al. Patterns of reduced cortical thickness in late-life depression and relationship to psychotherapeutic response. Am J Geriatr Psychiatry 2013; 21: 794-802.

41 Phillips JL, Batten LA, Tremblay P, Aldosary F, Blier P. A prospective, longitudinal study of the effect of remission on cortical thickness and hippocampal volume in patients with treatment-resistant depression. Int J Neuropsychopharmacol 2015; 18: pyv037.

42 Frodl T, Koutsouleri N, Bottlender R, Born C, Jäger M, Scupin I et al. Depressionrelated variation in brain morphology over 3 years. Arch Gen Psychiatry 2013; 65 : 1156-1165.

43 Coryell W, Nopoulos P, Drevets W, Wilson T, Andreasen N. Subgenual prefrontal cortex volumes in major depressive disorder and schizophrenia: diagnostic specificity and prognostic implications. Am J Psychiatry 2005; 162: 1706-1712.

44 Lim HK, Jung WS, Ahn KJ, Won WY, Hahn C, Lee SY et al. Regional cortical thickness and subcortical volume changes are associated with cognitive impairments in the drug-naive patients with late-onset depression. Neuropsychopharmacology 2012; 37: 838-849.

45 Whittle S, Lichter R, Dennison M, Vijayakumar N, Schwartz O, Byrne ML et al. Structural brain development and depression onset during adolescence: a prospective longitudinal study. Am J Psychiatry 2014; 171: 564-571.

46 Storsve AB, Fjell AM, Tamnes CK, Westlye LT, Overbye K, Aasland HW et al. Differential longitudinal changes in cortical thickness, surface area and volume across the adult life span: regions of accelerating and decelerating change. J Neurosci 2014; 34: 8488-8498.

47 Wierenga LM, Langen M, Oranje B, Durston S. Unique developmental trajectories of cortical thickness and surface area. Neuroimage 2014; 87: 120-126.
48 Jernigan TL, Brown T, Bartsch H, Dale AM. Toward an integrative science of the developing human mind and brain: focus on the developing cortex. Dev Cogn Neurosci 2016; 18: 2-11.

49 Amlien IK, Fjell AM, Tamnes CK, Grydeland H, Krogsrud SK, Chaplin TA et al. Organizing principles of human cortical development-thickness and area from 4 to 30 years: insights from comparative primate neuroanatomy. Cereb Cortex 2014; 26: 257-267.

50 Rakic P. A small step for the cell, a giant leap for mankind: a hypothesis of neocortical expansion during evolution. Trends Neurosci 1995; 18: 383-388.

51 Anderson BJ. Plasticity of gray matter volume: the cellular and synaptic plasticity that underlies volumetric change. Dev Psychobiol 2011; 53: 456-465.

52 Newman E, Thompson WK, Bartsch H, Hagler DJ, Chen C-H, Brown $\pi$ et al. Anxiety is related to indices of cortical maturation in typically developing children and adolescents. Brain Struct Funct 2015; e-pub ahead of print 17 July 2015; doi: 10.1007/s00429-015-1085-9.

53 Kelly PA, Viding E, Wallace GL, Schaer M, De Brito SA, Robustelli B et al. Cortical thickness, surface area, and gyrification abnormalities in children exposed to maltreatment: neural markers of vulnerability? Biol Psychiatry 2013; 74: 845-852.

54 Hodel AS, Hunt RH, Cowell RA, Van Den Heuvel SE, Gunnar MR, Thomas KM Duration of early adversity and structural brain development in postinstitutionalized adolescents. Neuroimage 2015; 105: 112-119.

55 Fairchild G, Toschi N, Hagan CC, Goodyer IM, Calder AJ, Passamonti L. Cortical thickness, surface area, and folding alterations in male youths with conduct disorder and varying levels of callous-unemotional traits. Neurolmage Clin 2015; 8: 253-260.

56 Wilson S, Vaidyanathan U, Miller MB, McGue M, lacono WG. Premorbid risk factors for major depressive disorder: Are they associated with early onset and recurrent course? Dev Psychopathol 2014; 26: 1477-1493.

57 Hill RM, Pettit JW, Lewinsohn PM, Seeley JR, Klein DN. Escalation to major depressive disorder among adolescents with subthreshold depressive symptoms: evidence of distinct subgroups at risk. J Affect Disord 2014; 158: 133-138.

58 Kambeitz-llankovic L, Meisenzahl EM, Cabral C, von Saldern S, Von, Kambeitz J, Falkai $\mathrm{P}$ et al. Prediction of outcome in the psychosis prodrome using neuroanatomical pattern classification. Schizophr Res 2015.

59 Im K, Lee J, Lyttelton O, Kim SH, Evans AC, Kim SI. Brain size and cortical structure in the adult human brain. Cereb Cortex 2008; 18: 2181-2191.

60 Fossati P, Radtchenko A, Boyer P. Neuroplasticity: from MRI to depressive symptoms. Eur Neuropsychopharmacol 2004; 14: S503-S510.

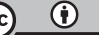

This work is licensed under a Creative Commons Attribution 4.0 International License. The images or other third party material in this article are included in the article's Creative Commons license, unless indicated otherwise in the credit line; if the material is not included under the Creative Commons license, users will need to obtain permission from the license holder to reproduce the material. To view a copy of this license, visit http://creativecommons.org/licenses/ by/4.0/

Supplementary Information accompanies the paper on the Molecular Psychiatry website (http://www.nature.com/mp) 